\title{
Study on Map Knowledge Domains of Transgenic Maize Based on Citespace
}

\author{
Xin-Tong Li, Dan-Hui Mao, Jing-Min Cheng* \\ School of Management, Shanxi Medical University, Taiyuan, China \\ Email address: \\ ZnCu_li@163.com (Xin-Tong Li), 784581223@qq.com (Dan-Hui Mao),72-87@163.com (Jing-Min Cheng) \\ *Corresponding author
}

To cite this article:

Xin-Tong Li, Dan-Hui Mao, Jing-Min Cheng. Study on Map Knowledge Domains of Transgenic Maize Based on Citespace. American Journal of Agriculture and Forestry. Vol. 6, No. 3, 2018, pp. 44-49. doi: 10.11648/j.ajaf.20180603.12

Received: April 1, 2018; Accepted: April 16, 2018; Published: May 18, 2018

\begin{abstract}
To analyze the hotspots and trend of the study on global transgenic maize, the paper collected literature on transgenic maize from 2001 to 2018 which are included in the core journals in the database of Web science as samples and used Citespace for visual analysis of mapping knowledge domain of studies on transgenic maize, based on the bibliometric method. As a result, 3546 pieces of documents have been sifted out from 3557 pieces of documents which have been retrieved according to the search strategy, the literature are in a stable situation and show a tendency of increase. The paper analyzed the scientific knowledge graphs on co-authors analysis network, co-institutions analysis network, author co-citation analysis network, citation analysis network and keyword co-appearance network by using Citespace software. Therefore, in cluster analysis, it is found that in the study on transgenic maize, Chinese scholars and institutions hold an advantage in quantity while the citation of foreigners'literature is higher than Chinese. Besides, the focus of global research is mainly about bacillus thuringiensis, arabidopsis thaliana, protein, gene expression, transformation, resistant, etc.
\end{abstract}

Keywords: Transgetic Maize, Citespace, Scientific Knowledge Graph, Hotspot, Trend

\section{Introduction}

As one of the five major cereal crops, corn occupies an important position in the world's agricultural production and plays an important role in the production of food and feed for all countries. Genetically modified food technology offers a efficient and low-cost solution to meet current and future challenges in food. According to statistics, the global planting area of genetically modified maize has reached 185.1 million square hectometer in 2016, and it is 110 times more than the 1.7 million square hectometer in 1996 [1]. Between 2007 and 2016, the top five countries in terms of global GM crop acreage were the United States, Brazil, Argentina, Canada, and India. The United States Department of Agriculture (USDA) statistics show that more than $90 \%$ of the soybeans planted in various states in the United States were genetically modified variety in 2016 [2]. In recent years, maize has been widely studied by scientists around the world as the main research object of transgenic technology. Research results have been applied to the application of transgenic technology, including resistance to maize borer, root insect pests and herbicides, and improvement of maize nutritional quality. This studies compensate for the limitations of traditional breeding methods in maize genetic improvement.

Since the advent of genetically modified corn, although some of its improved traits can meet people's requirements, its safety is still controversial. The safety issue of genetically modified corn mainly revolves around the safety of environment and food. The environmental safety of GM maize involve two parts, including the impact on soil ecosystems and gene drift. After many years of different experimental research, scientists have reached the basic and consistent conclusion that the consumption of genetically modified corn is safe, and there is almost no extreme case of abuse. But the public's confidence of genetically modified foods or plants still requires the efforts of more researchers. In addition, some people also hold opposing views that genetically modified foods are unsafe for human consumption, sometimes fuelled by criticisms based on little or no firm evidence [3]. Fifteen years ago, genetically modified (GM) crops has spread to the traditional corn 
landrace in Mexico, and it triggered a global discussionon about the scale and significance of the origin and the genetic diversity center of GM crops [4].

The use of bibliometrics to analyze andpredict the development trend of disciplines has become a method of analysis of development trends in various disciplines. José M analyzed bibliographic coupling, citation and co-citation analysis, co-authorship, and co-occurrence of keywords. The results underline the significant growth of the journal through time and its international diversity having publications from countries all over the world [5]. Bendegul Okumus examined the evolution of food and gastronomy research in hospitality and tourism (H \& T) in the 40 years between 1976 and 2016, they found although international and national collaboration of food-related management, finance, and marketing topics have been growing among different institutions in the $\mathrm{H} \& \mathrm{~T}$ field, only a limited number of articles seem to address food and gastronomy themes [6]. Wu S R [7] used the Chinese Academic Journal Network Publication Library as a data source to search a total of 587 articles. Based on bibliometrics methods, statistics were taken from output time, source journals, authors, issuing agencies, funds, and downloads and cited frequency. The analysis shows that research in the field of GM maize is receiving more and more attention from domestic scholars and is in an accelerated development stage. As a rapidly developing discipline, GM crop research currently lacks related research on the overall development trend of global transgenic research in recent years. China has a large scale in the overall scale of GM crop research. However, there is still much room for improvement in the overall scientific research level. Therefore, researchers should understand the global GM maize research field, and fully grasp the development history, the main thread, and the frontline trend of GM maize research. It is very necessary. This study analyzes the overview of the field of genetically modified maize research from 2001 to 2018 in order to provide scientific support for scientific researchers to better conduct scientific decisions for research and scientific management departments.

\section{Materials and Methods}

\subsection{Materials}

Web of Science is developed by the Thomson Scientific from America based on Web, including more than 8000 kinds of one of the most influential journals in the world, after experts' evaluation of quality of journals.

This paper completed data analysis, based on literature of "transgenic maize" which are found on the core journals of Web of Science. Specific search strategy is "subject = ('transgenic maize')", meanwhile, the range of time is set by "2001-2018", and the retrieval time is set by March 24, 2018. Totally searched 3557 literature, after screening for 3546. Citations saved for other format file, each topic including author, organization, keywords and references and other information.

\subsection{Methods}

Map Knowledge Domains is a combination of citation analysis with data and information visualization. Citespace is citation visualization software which analyzes the potential knowledge in scientific analysis and is developed under the background of scientometrics, big data and visualization of information.

Using Citespace to load normalized citations into calculate, the range of time is set as " 2001-2018", and time slice is set as 1 . Selecting the method of pathfinder in order to reduce the network density and improving the readability of the network. Firstly, screen out repeated citations. Secondly, choose different themes, including the authors, institutions, keywords, cited reference, cited authors, then choose clustering analysis, and finally resize the sizes of individual nodes, the threshold value. Thus it completes the Map Knowledge Domains.

\subsection{Posting}

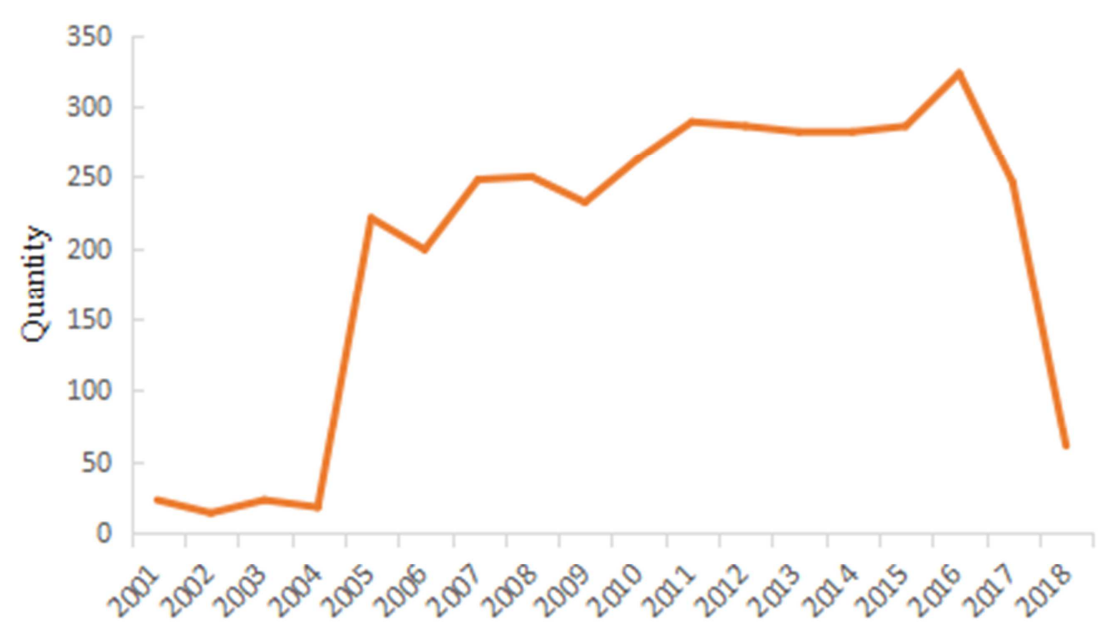

Figure 1. Posting of Transgenic Maize from 2001 to 2018. 
A statistical chart about the publication of transgenic maize clearly shows the overall situation of study. As is shown in figure 1, from 2001 to 2018, the global study on transgenic maize is keeping steady, published roughly 200 to 300 papers in each year and has a rising trend. Thus, the heat on the study of transgenic maize is relatively stable, and the study has already formed a certain scale and mode while the attention on this study is also increasing.

\section{Collaboration Network Analysis}

Citespace provides three levels of study, including coauthors, co-institutions, co- countries/ regions; the size of node size represents the number of published papers by individual authors, institutions, countries or regions [8]. This paper analyzes the cooperation network of transgenic maize.

\subsection{Co-authors Analysis}

Analyzing the network of cooperation between authors can intuitively find the important authors in the field of transgenic maize. Using clustering function analysis citations from 2001 to 2018, the threshold is set to 20, shown in figure 2. As shown, Romeis J, writing 47 papers, is the leading figure in the study of transgenic maize, followed by Liu Y, Huang FN, Hibbard BE, Christou P. Besides, the Chinese researchers in this study have advantage of quantity. In addition, various researchers maintained close relate from complex lines, shown that the study of transgenic maize have become interchanged and cooperated normally.

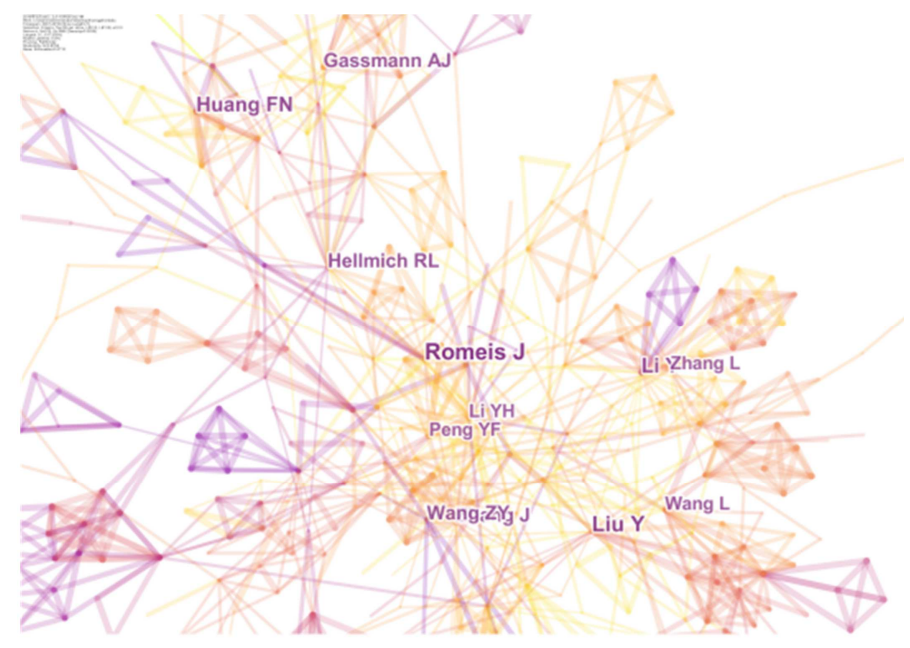

Figure 2. Co-authors Analysis Network.

\subsection{Co-institutions Analysis}

By analyzing the network of cooperation between institutions, it can intuitively find the important institutions in the field of transgenic maize. Using clustering function analysis citations from 2001 to 2018 , the threshold is set as 55, shown in figure 3 .
As shown in table 1, among the top 10 in the study of transgenic maize from 2001 to 2018, China holds amost half of the institutions, which is consistent with the above analysis results of important authors. It explains that China plays an important role in the study of transgenic maize again.

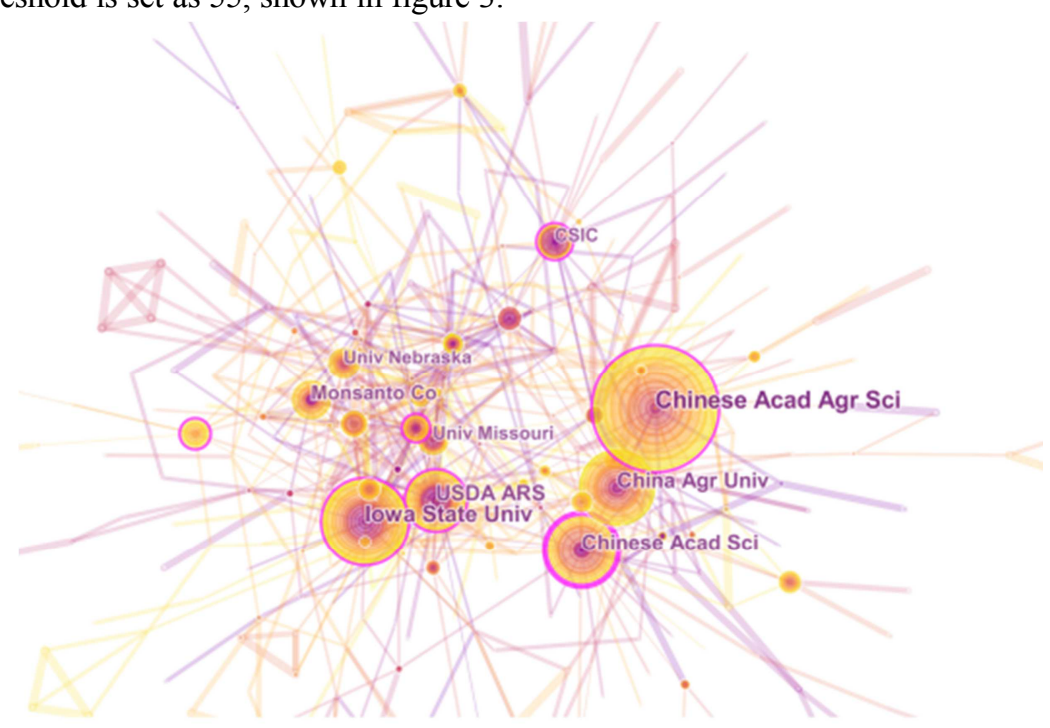

Figure 3. Co-institutions Analysis Network. 
Table 1. Post on top 10 of institutions.

\begin{tabular}{llllllll}
\hline No. & F & Centrality & Institution & No. & F & Centrality & Institution \\
\hline 1 & 180 & 0.12 & Chinese Acad Agr Sci & 6 & 73 & 0.03 & Monsanto Co \\
2 & 129 & 0.16 & Iowa State Univ & 7 & 64 & 0.08 & Univ Nebraska \\
3 & 120 & 0.06 & China Agr Univ & 8 & 63 & 0.13 & CSIC \\
4 & 105 & 0.26 & Chinese Acad Sci & 9 & 55 & 0.08 & Univ Missouri \\
5 & 102 & 0.18 & USDA ARS & 10 & 53 & 0.1 & Univ Illinois \\
\hline
\end{tabular}

\section{Co-citation Network Analysis}

Citation analysis is the method of information measurement that uses mathematical and statistical methods to compare, induct, abstract and generalize, analyses the phenomenon of cited or cite, such as scientific journals, papers, authors, to reveal the quantitative characters and inherent laws [9-10]. This paper mainly does citation analyzes through the author co-citation analysis and literature co-citaion.

By analyzing the situation of citation, it can intuitively find that the of high quality articles in the study of transgenic maize and its development of history and forefront. Using clustering function analysis citations from 2001 to 2018, the threshold is set to 55, shown in figure 4. It shows that there is little difference among the size of nodes, explaining situation of cited is similar located in the top. Among them, the articles written by ROMEIS J, published in 2006 and 2008, were cited 91 times and 65 times respectively. It shows that the number of articles written by ROMEIS $\mathrm{J}$ is large and the quality is also very high. Using clustering function analysis citations from 2001 to 2018 , the threshold is set to 125 , shown in figure 5. Among these authors, the largest amount of cited is SAMBROOK J, cited 299 times. By contrast, in term of the number of the important authors and institutions, China has its advantage. In the analysis of cited, this advantage, however, does not display.

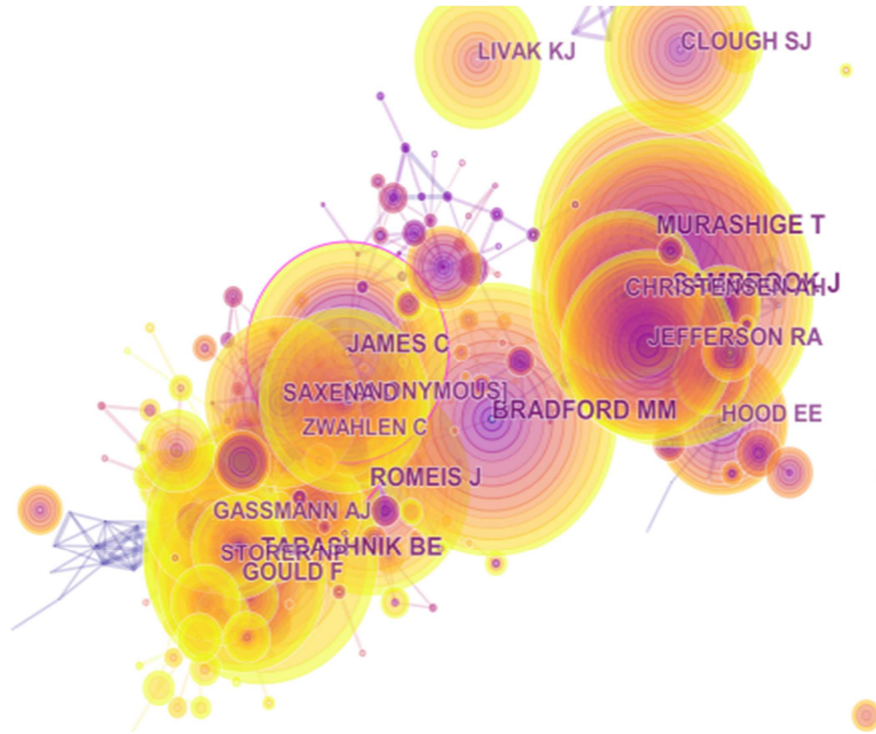

Figure 4. Citation analysis Network.

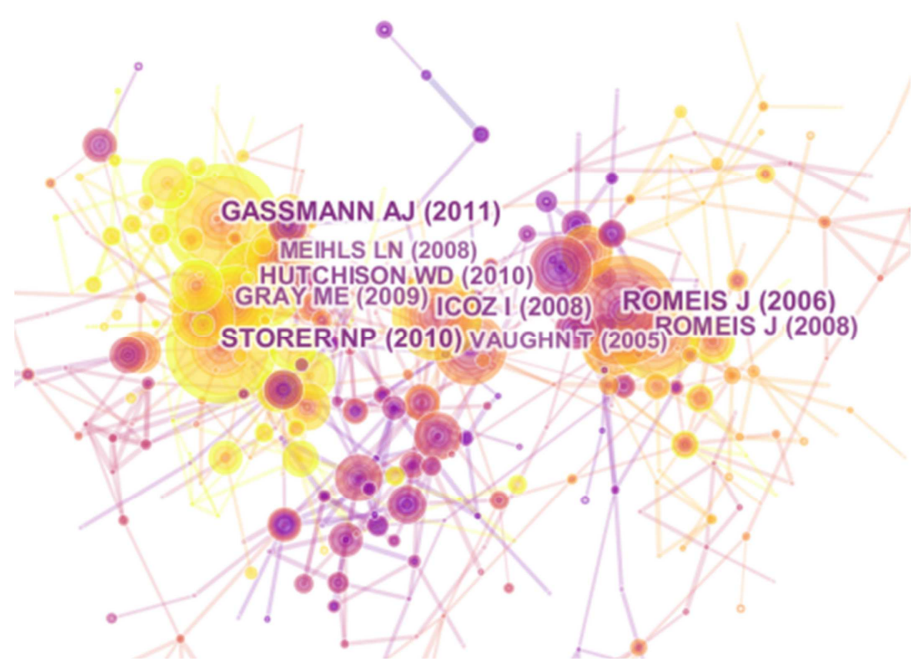

Figure 5. Author co-citation Analysis Network. 


\section{Co-appearance Network}

Taking keywords as nodes to analyze, it is found that a higher frequency indicates a bigger font. Using clustering function analysis citations from 2001 to 2018, the threshold is set to 270 , shown in figure 6 . Behind the high heat of keywords on top, the uses of following became smooth and steady. Table 2, the situation of uses of keywords in transgenic maize from 2001 to 2018, shows top 20. "maize", "zea may", and "corn" are synonymous with the term of "corn", "transgenic maize", "transgenic corn", and "bt maize" are synonymous with the term of "transgenic maize", "plant" and "crop" are synonymous with "crop". Thus, the study in transgenic maize from 2001 to 2018 mainly revolves in these aspects, such as bacillus thuringiensis, arabidopsis thaliana, protein, gene expression, transformation, resistant.

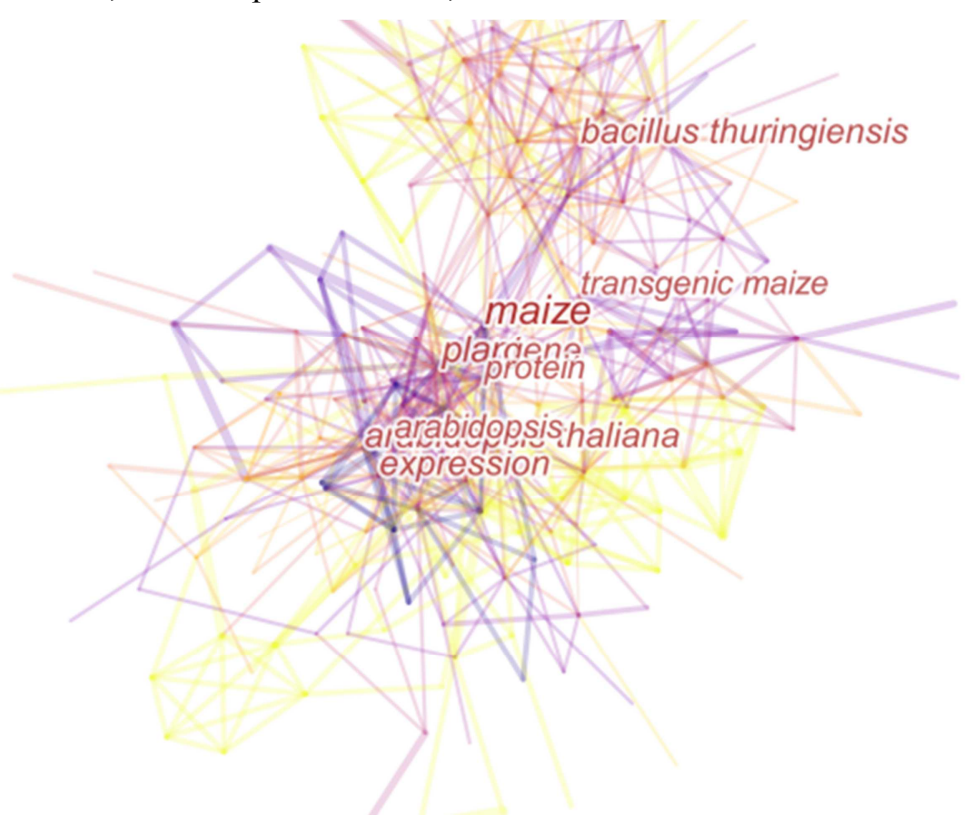

Figure 6. Keyword co-appearance Network.

Table 2. Keywords on top 20.

\begin{tabular}{|c|c|c|c|c|c|c|c|}
\hline No. & $\mathbf{F}$ & Centrality & Keyword & No. & $\mathbf{F}$ & Centrality & Keyword \\
\hline 1 & 1172 & 0.17 & maize & 11 & 268 & 0.08 & rice \\
\hline 2 & 621 & 0.12 & plant & 12 & 219 & 0.17 & gene expression \\
\hline 3 & 455 & 0.19 & expression & 13 & 211 & 0.06 & zea may \\
\hline 4 & 419 & 0.15 & bacillus thuringiensis & 14 & 206 & 0.26 & transformation \\
\hline 5 & 377 & 0.07 & arabidopsis thaliana & 15 & 204 & 0.13 & resistance \\
\hline 6 & 356 & 0.08 & gene & 16 & 202 & 0.08 & bt maize \\
\hline 7 & 297 & 0.11 & transgenic maize & 17 & 196 & 0.16 & corn \\
\hline 9 & 284 & 0.12 & protein & 19 & 133 & 0.06 & transgenic rice \\
\hline 10 & 269 & 0.1 & transgenic plant & 20 & 133 & 0.09 & transgenic corn \\
\hline
\end{tabular}

\section{Conclusions}

This paper mainly uses Citespace, a scientific metrology software, analyzing citation data in the corn of Web of Science about transgenic maize from 2001 to 2018, drawing Map Knowledge Domains. After analysis, this paper found that Romeis $\mathrm{J}$ is one of the main scholars in the study of trandgenic maize. In addition, the researchers and institutions in China have advantage in quantity. Through the analysis in the situation of cited about literature and authors cited, this paper found that as for the amount of literature being cited, the foreign ones are higher than those of the Chinese. Therefore China should improve the quality of the papers. At the same time, hotspots in recent years mainly converge on bacillus thuringiensis, arabidopsis thaliana, protein, gene expression, transformation, resistant. As a hot topic in the food safety, transgenic maize has gained a multi-dimensional debate [11]. Capturing the latest development in research on transgenic maize, China should focus on the global hotspot of transgenic maize, expand international cooperation and consistently promote further development of the study.

\section{Acknowledgements}

This work is supported by the Fund (grant no.20160420086) hosted by Jing-Min Cheng for the research on the development status and innovation mechanism of biomedicine industry in Shanxi province. 


\section{References}

[1] Cilve James. Global Development of Commercialization of Genetically Modified Crops in 2016 [J]. China Biotechnology, 2017, 37(4):1-8. (In Chinese)

[2] USDA Data. Adoption of genetically engineered crops in the U.S. retrieved [EB/OL]. https://www.ers.usda.gov/data products/adoption-of-genetically-engineered-crops-in-theus.aspx, 2017.

[3] Lee TH; Ho HK; Leung TF. Genetically modified foods and allergy [J]. Hong Kong Med Journal, 2017, 23(3):291-295.

[4] Sarah Z. Agapito-Tenfen;Fern Wickson. Challenges for transgene detection in landraces and wild relatives: learning from 15 years of debate over GM maize in Mexico [J]. Biodiversity and Conservation, 2018, 27(3):539-566.

[5] José M. Merigó, Witold Pedrycz, Richard Weber, et al. Fifty years of Information Sciences: A bibliometric overview [J]. Information Sciences, 2018, 432:245-268.

[6] Bendegul Okumus, Mehmet Ali Koseoglu, Forest Ma. Food and gastronomy research in tourism and hospitality: A bibliometric analysis [J]. International Journal of Hospitality Management, 2018, 73:64-74.

[7] Wu Shirong. Bibliometric Analysis of Genetically Modified Maize Research in China [J]. Journal of Library and Information Sciences in Agriculture, 2014, 26(4):50-53. (In Chinese)

[8] Wei-jun Wang, Guo-pei Cai. Method and Application of Information Analysis. Beijing Jiaotong University Press, 2010, 5-10. (In Chinese)

[9] Chen C. Cite Space II: Detecting and Visualizing Emerging Trends and Transient Patterns in Scientific Literature. Journal of the American Society for Information Science and Technology2006;3:359-377.

[10] Jie Li, Chao-mei Chen. Citespace: Text Mining and Visualization in Scientific Literature. Capital University of Economics and Business Press, 2016, 180-182. (In Chinese)

[11] Jing-min Cheng. Study on Pre-warning System of Food Safety. The Economics Daily Press, 2014, 13-16. (In Chinese) 